\title{
BERRY CURVATURE IN GRAPHENE: A NEW APPROACH
}

\author{
Pierre Gosselin \\ Institut Fourier, UMR 5582 CNRS-UJF, UFR de Mathématiques, \\ Université Grenoble I, \\ BP74, 38402 Saint Martin d'Hères, Cedex, \\ France.
}

\author{
Alain Bérard, and Hervé Mohrbach \\ 2843 , \\ Université Paul Verlaine-Metz, \\ 57078 Metz Cedex 3, \\ France. \\ Subir Ghosh \\ Physics and Applied Mathematics Unit, \\ Indian Statistical Institute, \\ 203 B. T. Road, Calcutta 700108, \\ India.
}

Laboratoire de Physique Moléculaire et des Collisions, ICPMB-FR CNRS 


\begin{abstract}
:
In the present paper we have directly computed the Berry curvature terms relevant for Graphene in the presence of an inhomogeneous lattice distortion. We have employed the generalized Foldy Wouthuysen framework, developed by some of us [4, 5, 6]. We show that a non-constant lattice distortion leads to a valley-orbit coupling which is responsible to a valley-Hall effect. This is similar to the valley-Hall effect induced by an electric field proposed in [14] and is the analogue of the spin-Hall effect in semiconductors [16, 17]. Our general expressions for Berry curvature, for the special case of homogeneous distortion, reduce to the previously obtained results [14. We also discuss the Berry phase in the quantization of cyclotron motion.
\end{abstract}

Keywords: Graphene, Foldy Wouthuysen, Berry curvature, anomalous velocity

PACS Numbers: 


\section{Introduction}

The latest avatar of crystalline carbon - Graphene [1] - has evolved into a meeting ground of Particle and Condensed Matter physicists. The reason is that under certain conditions the electronic excitations in graphene behave like massless relativistic fermions moving with (energy independent) Fermi velocity $v_{F} \approx 10^{6}$ $\mathrm{m} / \mathrm{s}$, that plays the role of the velocity of light 2 . The honeycomb lattice of graphene is made up of two triangular lattices $(A$ and $B)$ and the hexagonal Brillouin zone has two distinct and degenerate Dirac points or valleys $\left(K_{+}\right.$and $K_{-}$) where the conduction and valence bands meet. In the undoped case the chemical potential passes through the Dirac points so that the valence band is filled whereas the conduction band is empty. In the Tight Binding Approximation, the dynamical equations of motion for the planar electrons (with spin not taken in to account) for the above four types can be arranged such that one has a four-component Dirac equation at hand. This truly remarkable mapping has led to the predictions that a number of Particle Physics phenomena, such as Klein tunnelling, Andreev reflection among others, can be (and have been) observed in graphene [1].

However, from the present literature on graphene, it can be generally observed that explicit computations in the context of graphene are not performed in the four component Dirac form. In the present paper we provide a new approach where explicit use has been made of the Dirac framework. We exploit a generalized Foldy-Wuthouysen (FW) formalism [3] used for four component Dirac Hamiltonian (the reason to use the four component Dirac form is thus mainly technical), recently developed by some of us [4, 5, 6], where one can study the Dirac Hamiltonian with very general type of interaction. The result comes as a series in powers of $\hbar$. It is very important to stress that in the present work, we have also formulated a method of projection that can isolate the single valley effects (via two component Pauli spinors) from the Dirac spinor expressions and hence our method can be used to study single valley and inter-valley interaction effects.

The method relies heavily on the appearance of intrinsic Berry gauge potentials 7,8, that induce a non-commutative geometry (Berry curvature) in the electron phase space which subsequently affects the particle dynamics [9] (see also [10] for a review). In the $\mathrm{FW}$ formalism, in order to properly identify the single particle operators (see for example [11]), one needs to perform the same unitary transformation $U$ on all the operators (position $\mathbf{r}$, momentum $\mathbf{p}$ etc.), that (partially) diagonalizes the Hamiltonian $H$,

$$
U H U^{+}=E ; \quad \mathbf{r}=U \mathbf{r} U^{+}=\mathbf{R}+i \hbar U \nabla U^{+} \equiv \mathbf{R}+\hbar \mathcal{A}^{R}
$$

Here $E$ denotes the diagonal energy eigenvalue matrix and $\mathcal{A}^{R}$ is the Berry potential. One can physically motivate the introduction of $\mathbf{r}$ by noting that in a Hamiltonian framework it yields the correct expression for the velocity. Incidentally the configuration space $\mathbf{r}$ (as well as the full phase space) turns out to be noncommutative. (The earliest example of a noncommutative space 
(or phase space) was provided in [12].) This is the "algebraic" reason for the emergence of Berry phase.

On the other hand, intuitively the appearance of Berry phase effects is very natural in FW formalism. The Berry potential is induced by the electron spin coordinates which are treated as "fast" variables as compared to the position coordinates which are considered as "slow" degrees of freedom. The same phenomena was discovered earlier [13] when molecular dynamics was studied in the Born-Oppenheimer approximation.

The advantage of our method is that one can study all these interactions in a unified framework. In fact, for a constant distortion and for zero magnetic field we recover the results of [14. But obviously more interesting is the new results that we obtain for non-constant lattice distortion, which is a new valley-orbit coupling due to the spatial variation of the mass gap induced by the lattice distortion. This coupling is similar to the valley-orbit coupling in the presence of an external electric field and in turn contributes to the valley-Hall effect first predicted in 14.

In this paper we also consider the semiclassical quantization of the cyclotron orbit for an electron in a constant magnetic field and for a constant mass. In particular we show the important role played by the valley magnetic moment in the determination of the Landau levels.

The paper is organized as follows: In section $\mathbf{2}$ we discuss the technicalities, construct the (non-commutative) covariant position and momentum operators, identify the Berry potentials and compute the semi-classical energy spectrum, in a generic framework. In Section 3 we apply the scheme to graphene. In Section 3 we focus on effects that result from the variable lattice distortion. Section 4 deals with effects due to external electromagnetic fields. Section $\mathbf{5}$ is devoted to discussions.

\section{Covariant phase space and Berry potentials}

We will explain our formalism through the concrete example of graphene. Around the $K_{+}$and $K_{-}$points the energy spectrum is linear and the dispersion relation can be approximated as $E_{ \pm}(K)=\hbar v_{F}\left|K-K_{ \pm}\right|$. In the following $v_{F}$ is set to unity so that the effective Hamiltonian near both valleys in the presence of a distorted lattice, induced by a real bosonic field $\Delta(\mathbf{R})$ and in the presence of an external electric potential $V(\mathbf{R})$ can be written as

$$
H(\mathbf{P}, \mathbf{R})=\alpha . \mathbf{P}+\beta \Delta(\mathbf{R})-e V(\mathbf{R}) .
$$

where $e>0$ so that the charge of the electron is $-e$. In this expression the energy is written in terms of the momentum $\mathbf{P}=\hbar \mathbf{K}$ in order that $\hbar$ appears explicitly during the semiclassical diagonalization procedure described below. We have also used the following representation for the Dirac matrices

$$
\alpha_{x}=\left(\begin{array}{cc}
\sigma_{x} & 0 \\
0 & -\sigma_{x}
\end{array}\right) \quad \alpha_{y}=\left(\begin{array}{cc}
\sigma_{y} & 0 \\
0 & \sigma_{y}
\end{array}\right) \quad \beta=\left(\begin{array}{cc}
\sigma_{z} & 0 \\
0 & \sigma_{z}
\end{array}\right)
$$


where $\sigma_{i}$ are Pauli matrices. The dynamical operators $\mathbf{P}$ and $\mathbf{R}$ have components satisfying the canonical rule $\left[R_{i}, P_{j}\right]=i \hbar \delta_{i j}$. We keep the $3+1$-dimensional representation with the understanding that the components in the $z$-direction are null $R_{z}=P_{z}=0$ because graphene is a planar 2-dimensional system.

Since we are interested in obtaining a semi-classical solution, we first diagonalize the "classical" Hamiltonian (1) (whose arguments are not operators but classical commuting variables noted $(\widetilde{\mathbf{P}}, \widetilde{\mathbf{R}}))$ by a formal FW unitary transformation $U_{0}$ and later reinsert the operators back. The $U_{0}$ in question is a conventional FW matrix,

$$
U_{0}(\widetilde{\mathbf{P}}, \widetilde{\mathbf{R}})=\frac{E+\Delta(\widetilde{\mathbf{R}})+\beta \alpha . \widetilde{\mathbf{P}}}{\sqrt{2 E(E+\Delta(\widetilde{\mathbf{R}}))}}
$$

such that the diagonal form of Hamiltonian is,

$$
U_{0} H U_{0}^{+}=\varepsilon_{0}(\widetilde{\mathbf{P}}, \widetilde{\mathbf{R}})=\beta \sqrt{\widetilde{\mathbf{P}}^{2}+\Delta(\widetilde{\mathbf{R}})^{2}}-e V(\widetilde{\mathbf{R}}) .
$$

where $E=\sqrt{\widetilde{\mathbf{P}}^{2}+\Delta(\widetilde{\mathbf{R}})^{2}}$. After this "classical diagonalization" the reintroduction of the quantum operators $\mathbf{P}$ and $\mathbf{R}$ leads to the following semiclassical diagonal Hamiltonian $H_{D}[\underline{6}$,

$\varepsilon(\mathbf{p}, \mathbf{r}) \simeq \varepsilon_{0}(\mathbf{p}, \mathbf{r})+\frac{i \hbar}{2} \mathcal{P}_{+}\left[\left[\varepsilon_{0}(\mathbf{p}, \mathbf{r}), \mathcal{A}^{R_{l}}\right] \mathcal{A}^{P_{l}}-\left[\varepsilon_{0}(\mathbf{p}, \mathbf{r}), \mathcal{A}^{P_{l}}\right] \mathcal{A}^{R_{l}}\right]+O\left(\hbar^{2}\right)$.

In the above $\mathcal{P}_{+}$denotes projection on the diagonal subspace and

$$
\varepsilon_{0}(\mathbf{p}, \mathbf{r})=\beta \sqrt{\mathbf{p}^{2}+\Delta(\mathbf{r})^{2}}-e V(\mathbf{r}) .
$$

In this expression the canonical operators $(\mathbf{R}, \mathbf{P})$ have been replaced by the covariant ones,

$$
\begin{aligned}
& \mathbf{r}=\mathbf{R}+\hbar A^{\mathbf{R}} \\
& \mathbf{p}=\mathbf{P}+\hbar A^{\mathbf{P}}
\end{aligned}
$$

where we have also introduced the so called Berry connections

$$
A^{\mathbf{R}}=\mathcal{P}_{+}\left[\mathcal{A}^{\mathbf{R}}\right] ; A^{\mathbf{P}}=\mathcal{P}_{+}\left[\mathcal{A}^{\mathbf{P}}\right],
$$

defined as the projection on the diagonal of $\mathcal{A}^{\mathbf{R}}=i\left[U_{0} \nabla_{\mathbf{P}} U_{0}^{+}\right], \mathcal{A}^{\mathbf{P}}=-i\left[U_{0} \nabla_{\mathbf{R}} U_{0}^{+}\right]$. Here $U_{0}$ denotes the matrix $U_{0}(\mathbf{P}, \mathbf{R})$ that is the matrix $U_{0}$ where now parameters have been replaced by operators.

Notice the reason for projecting on the diagonal is same as in the conventional FW case [1] where this ensures that positive energy solutions do not 
get mixed up with negative energy solutions. In the present Condensed Matter scenario, these correspond to conduction band and valence band.

From $U_{0}$ we can now deduce the following covariant coordinate and momentum

$$
\mathbf{r}=\mathbf{R}-\hbar \frac{\mathbf{e}_{z} \times \mathbf{P}}{2 E(E+\Delta)} \Sigma_{z}, \quad \mathbf{p}=\mathbf{P},
$$

where $\mathbf{e}_{z}$ is the unit vector in the $z$-direction and $\Sigma_{z}=\left(\begin{array}{cc}\sigma_{z} & 0 \\ 0 & -\sigma_{z}\end{array}\right)$. Notice that the momenta remain unchanged. This will be changed in the presence of an external magnetic field.

From the general expression (5) we compute the full diagonal quantum Hamiltonian,

$$
\varepsilon=\beta E-\frac{\hbar}{2 E^{2}} \mathbf{e}_{z}\left(\mathbf{p} \times \nabla_{\mathbf{r}} \Delta(\mathbf{r})\right) \Sigma_{z}-e V(\mathbf{r})+O\left(\hbar^{2}\right)
$$

where now $E=\sqrt{\mathbf{p}^{2}+\Delta(\mathbf{r})^{2}}$. However, all this is in the $3+1$-dimensional framework and to be applicable to graphene we now need to reduce the model dimensionally. This we do in the next section. The coordinate and momentum operators $\mathbf{r}$ and $\mathbf{p}$ are covariant operators because the energy operator (9) expressed in terms of $\mathbf{r}$ and $\mathbf{p}$ is gauge independent (i.e. independent of Berry connection).

\section{Single valley Hamiltonian for graphene}

In case of graphene one generally considers the two valleys to be isolated and studies the electron behavior near one valley. This is achieved by keeping the components $(1,2)$ of the Hamiltonian for one valley and $(3,4)$ for the second valley. Therefore the 2 -bands valley energy can be indexed by $\tau= \pm 1$ such that :

$$
\varepsilon_{\tau}=\sigma_{z}\left(E-\tau \frac{\hbar}{2 E^{2}} \mathbf{e}_{z}\left(\mathbf{p} \times \nabla_{\mathbf{r}} \Delta(\mathbf{r})\right)\right)-e V(\mathbf{r})
$$

where the eigenvalues \pm 1 of $\sigma_{z}$ corresponds to the conduction and valence band energies of the valley $\tau$. The position operator also is now given by the projection of (8) on the components $(1,2)$,

$$
\mathbf{r}=\mathbf{R}-\tau \frac{\hbar \mathbf{e}_{z} \times \mathbf{P}}{2 E(E+\Delta)} \sigma_{z}
$$

Now and for the rest of the paper we consider the properties of an electron in a conduction band (the positive energy band of (10) ) of a valley, which means that the position operator also has to be projected on this subspace leading to the expression

$$
\mathbf{r}=\mathbf{R}-\tau \frac{\hbar \mathbf{e}_{z} \times \mathbf{P}}{2 E(E+\Delta)}
$$


From this expression of the valley dependent coordinate operator we deduce that the Algebra satisfied by the covariant dynamical variables is a non commutative one expressed in terms of Berry curvatures $\Theta_{i j}^{\zeta \eta}=\partial_{\zeta^{i}} A_{j}^{\eta}-\partial_{\eta^{i}} A_{j}^{\zeta}$ where $\zeta, \eta$ mean either $r$ or $p$,

$$
\begin{aligned}
{[x, y] } & =i \hbar^{2} \Theta_{x y}^{r r}=-i \hbar^{2} \frac{\tau \Delta}{2 E^{3}} \\
{\left[p_{i}, r_{j}\right] } & =-i \hbar \delta_{i j}+i \hbar^{2} \Theta_{i j}^{p r}=-i \hbar \delta_{i j}+\tau \frac{i \hbar^{2}}{2 E^{3}} \varepsilon^{j k 3} P_{k} \nabla_{R_{i}} \Delta \\
{\left[p_{x}, p_{y}\right] } & =i \hbar^{2} \Theta_{x y}^{p p}=0
\end{aligned}
$$

The Berry curvature $\Theta_{x y}^{r r}=-\frac{\tau \Delta}{2 E^{3}}$ obtained here is denoted in the rest of the paper simply by $\Theta$. We mention that the general expression in (13) reduces to that of [14] who considered valley Hall effect for a constant distortion $\Delta$. We, on the other hand, show that for the more general case of non-constant distortion, there are additional Berry curvature components of the mixed form $\Theta_{i j}^{p r}$. In the absence of a magnetic field the Berry curvature $\Theta_{x y}^{p p}$ is zero. interestingly, the energy can be rewritten in terms of the Berry curvature as $\varepsilon_{\tau}=E-e V(\mathbf{r})+$ $\hbar E\left(\Theta_{x x}^{p r}+\Theta_{y y}^{p r}\right)$.

At this level, it is interesting to express the conduction band energy in terms of the canonical coordinates $\mathbf{P}$ and $\mathbf{R}$ which reads

$\varepsilon_{\tau}=\sqrt{\mathbf{P}^{2}+\Delta(\mathbf{R})^{2}}-\frac{\hbar \tau}{2 E(\Delta+E)} \mathbf{P} \times\left[\left(\frac{2 \Delta+E}{E}\right) \nabla_{\mathbf{R}} \Delta-e \nabla_{\mathbf{R}} V\right] \cdot \mathbf{e}_{z}-e V(\mathbf{R})$

For a constant mass term $\Delta=c s t$, we observe a valley-orbit coupling term $\frac{\hbar \tau}{2 E(\Delta+E)} \mathbf{P} \times e \nabla_{\mathbf{R}} V$ which is of the same origin as that of the fully relativistic spin orbit coupling $\frac{\hbar}{2 E(m+E)} \sigma \cdot\left(\mathbf{P} \times e \nabla_{\mathbf{R}} V\right)$ in the Dirac equation [4] 15. In the literature this coupling is usually written in the non-relativistic limit only $\frac{\hbar}{4 m^{2}} \sigma .\left(\mathbf{P} \times e \nabla_{\mathbf{R}} V\right)$ because it comes out from the Foldy-Wouthuysen transformation which is an expansion in $1 / \mathrm{m}$. The semiclassical diagonalization allows one to consider the fully relativistic situation. Interestingly for a non constant mass term we observe an additional valley-orbit coupling $\hbar \tau\left(\frac{2 \Delta+E}{2 E^{2}(\Delta+E)}\right) \mathbf{P} \times \nabla_{\mathbf{R}} \Delta$. Therefore a variable mass term mimics an external electric field and can thus lead to similar effects as with a true electric field as discuss in next section. All this shows that the non-commutative position operator encodes through the Berry potential the spin or valley-orbit coupling. In particular, as discussed in [4, the dynamics of the canonical operators turn out to be different from that of the non-commutative ones.

\section{Effect of variable lattice distortion $\Delta(\mathbf{r})$}

As already stated, $\nabla_{\mathbf{R}} \Delta$ term induces an additional valley-orbit coupling. More precisely, the field $\Delta(\mathbf{R})$ is responsible for a valley-orbit coupling term $\sim \mathbf{P} \times$ $\nabla_{\mathbf{R}} \Delta$ similar to the valley-orbit coupling induced by the electric field $\sim \mathbf{P} \times \nabla_{\mathbf{R}} V$ 
which in turn is of the same nature as the spin-orbit coupling for Dirac electrons in the non-relativistic limit (Pauli term). Our results reveal that a defect of the lattice distortion can actually be interpreted as an effective electric field, which can be responsible of a mechanical force leading to a valley Hall effect as the electric field does [14. This is closely related to the spin Hall effect in semiconductors [16], [17]). This is a new observation. This clearly reminds us of the work of Bernevig and Zhang [18. who showed that shear strain in zincblende semiconductors such as GaAs induces a spin-orbit coupling term which mimics the usual spin-orbit in the presence of an electric field (this mechanism in 18, then leads to a quantum spin Hall effect, which is different from the effect considered here ).

Interestingly within our approach we can study the effect of the valley-orbit coupling in both "ultra-relativistic" $(\Delta<<P)$ and "non-relativistic" limits $(\Delta>>P)$.

In the regime of a large gap $\Delta>>P$, the energy becomes

$$
\varepsilon_{\tau} \approx \Delta(\mathbf{R})+\frac{\mathbf{P}^{2}}{2 \Delta(\mathbf{R})}-\frac{\hbar \tau}{4 \Delta(\mathbf{R})^{2}} \mathbf{P} \times\left[3 \nabla_{\mathbf{R}} \Delta(\mathbf{R})-e \nabla_{\mathbf{R}} V(\mathbf{R})\right] \cdot \mathbf{e}_{z}
$$

which shows the same behavior than the usual non-relativistic limit energy of the Dirac Hamiltonian. For a constant $\Delta$, the valley-orbit contribution is cancelled by the presence of the mass-gap term $4 \Delta^{2}$ which is large compared to electron momentum (this is similar to the mass term $4 m^{2} c^{2}$ in the Pauli term of nonrelativistic Dirac electron). But this coupling can be greatly enhanced in the presence of a very sharp variation of the lattice distortion $\Delta$. This effect could be much larger than the valley-orbit coupling in the presence of an electric field if $\nabla_{\mathbf{R}} \Delta>>e \nabla_{\mathbf{R}} V$. Such very localized lattice distortion defect might be built by adsorbing the graphene on two different substrates in contact creating in this way a line defect.

In the opposite regime $\Delta<<P$ we have

$$
\varepsilon_{\tau}=P-\frac{\hbar \tau}{2 P^{2}} \mathbf{P} \times\left[\nabla_{\mathbf{R}} \Delta-e \nabla_{\mathbf{R}} V\right] \cdot \mathbf{e}_{z}
$$

Here the valley-orbit coupling is independent of the magnitude of the distortion field and can also be greatly enhanced by a very sharp variation of the distortion $\Delta$.

Therefore in both cases, a large gradient of the lattice distortion will generate a bigger valley-orbit interaction than with the presence of an electric field. This coupling may in principle induce a valley-Hall effect very similar to the valleyHall effect considered in [14, the latter having an electric field and a uniform $\Delta$.

This valley-Hall effect is better seen in the equations of motion which are given in terms of the covariant operators. The above noncommutative algebra (13) leads to a non-trivial modification in the dynamics of an electron in a conduction band of a valley which can be written

$$
\dot{\mathbf{r}}=\nabla_{\mathbf{p}} \varepsilon\left(1-\hbar \Theta^{p r}\right)-\hbar \dot{\mathbf{p}} \times \boldsymbol{\Theta}, \quad \dot{\mathbf{p}}=-\nabla_{\mathbf{r}} \varepsilon\left(1-\hbar \Theta^{p r}\right)
$$


where we have defined the curvature vector $\boldsymbol{\Theta}=\Theta \mathbf{e}_{z}$ and $\nabla_{\mathbf{p}} \varepsilon\left(1-\hbar \Theta^{p r}\right)$ has the following meaning $\nabla_{p^{j}} \varepsilon\left(\delta_{i j}-\hbar \Theta_{i j}^{p r}\right)$.

In more details at the first order in $\hbar$ we obtain for the momentum dynamics

$$
\dot{\mathbf{p}}=-\frac{\Delta}{E} \nabla_{\mathbf{r}} \Delta+e \nabla_{\mathbf{r}} V
$$

such that the velocity reads:

$$
\dot{\mathbf{r}}=\frac{\mathbf{p}}{E}-\frac{\hbar \tau}{2 E^{3}}\left(\left(\frac{p^{2}+2 \Delta^{2}}{E}\right) \nabla_{\mathbf{r}} \Delta-\Delta e \nabla_{\mathbf{r}} V\right) \times \mathbf{e}_{z}
$$

Importantly, the lattice distortion $\Delta(\mathbf{R})$ induces an anomalous velocity $\dot{\mathbf{p}} \times \mathbf{\Theta}$ even in the absence of an electric field. Suppose that the distortion is in the $y-$ direction. Then we get a spontaneous (non-steady) current both in the longitudinal ( $y$-direction) and in the transverse $x$-direction due to the anomalous velocity. Then electrons will accumulate irrespective of their magnetic moment at the longitudinal edge. And a electric potential between the edges in the $y-$ direction can be measured. In the same manner, we will have a spontaneous separation of the electrons depending on the value of their magnetic moments and thus an accumulation in the transverse edges. A steady currents requires an electric field. If the mass-valley-orbit coupling is stronger than the electricvalley-orbit coupling, then the dominant transverse current is due to the lattice distortion and leads a transverse velocity $v_{x} \simeq \frac{-\hbar \tau}{2 E^{3}}\left(\frac{p^{2}+2 \Delta^{2}}{E}\right) \nabla_{y} \Delta$ which depends of the valley index but is charge independent. Therefore there will be a transverse current in the $x$-direction $j_{x}(\mathbf{r}) \simeq e \frac{\hbar \tau}{2} \int d \mathbf{p} g(\mathbf{p}, \mathbf{r})\left(\frac{p^{2}+2 \Delta^{2}}{E^{4}}\right) \nabla_{y} \Delta$ where $g(p, r)$ is the statistical distribution of carrier, leading to a Valley Hall effect (separation of the carrier depending of the valley). Here we considered the electron in a ballistic regime neglecting scattering with the impurities. If the the system is not connected to the leads along the $x$-direction, carriers of different valleys accumulate near the edges of the sample as discussed in [14. If the density of up and down magnetic moments are equal there will be not potential difference in the $x$-direction. To observe a transverse electric potential one needs to control the chemical potential of both species as suggested in [14.

We now consider the case of the graphene in an external electromagnetic field.

\section{Graphene in a magnetic field}

The magnetic field interaction is introduced via minimal coupling and the Dirac Hamiltonian becomes,

$$
H(\mathbf{P}, \mathbf{R})=\alpha . \Pi+\beta \Delta(\mathbf{R})+V(\mathbf{R})
$$

with $\boldsymbol{\Pi}=\mathbf{P}+e \mathbf{A}$ the covariant momentum. The semiclassical evolution of a Dirac electron in an electromagnetic field for a constant mass term was first derived in reference $[9$. For the generalization to a variable mass we use the general method of Hamiltonian diagonalization of [6]. 


\subsection{Hamiltonian diagonalization}

As shown in [6], the introduction of the gauge potential is a straightforward generalization of Eq. (10) and one obtains for the valley $\tau$ the following diagonal representation of the valley Hamiltonian for a carrier in the conduction band

$$
\varepsilon_{\tau}=\sqrt{\pi^{2}+\Delta(\mathbf{r})^{2}}-\tau \frac{\hbar}{2 E^{2}} \mathbf{e}_{z}(\pi \times \nabla \Delta(\mathbf{r}))+e \hbar E \Theta B_{z}-e V(\mathbf{r})
$$

with $\mathbf{r}=\mathbf{R}+\hbar A^{\mathbf{R}}, \pi=\mathbf{\Pi}-e \hbar A^{\mathbf{R}} \times \mathbf{B}$ and $\mathbf{B}=\nabla \times \mathbf{A}$. The covariant dynamical operators are explicitly given by

$$
\mathbf{r}=\mathbf{R}-\tau \frac{\hbar \mathbf{e}_{z} \times \boldsymbol{\Pi}}{2 E(E+\Delta)} \text { and } \pi=\boldsymbol{\Pi}+\tau \frac{e \hbar \boldsymbol{\Pi}}{2 E(E+\Delta)} B_{z}
$$

where $E=\sqrt{\pi^{2}+\Delta(\mathbf{r})^{2}}$. We note that in the presence of a magnetic field the momentum get a Berry connection a well. Therefore the new commutation relations are,

$$
\begin{aligned}
& {\left[r_{x}, r_{y}\right]=i \hbar^{2} \Theta=-i \hbar^{2} \frac{\tau \Delta}{2 E^{3}}} \\
& {\left[\pi_{i}, r_{j}\right]=-i \hbar \delta_{i j}-i e \hbar^{2} \Theta_{i l} \varepsilon^{l j 3} B_{z}-i \hbar^{2} \frac{\tau e}{2 E^{3}} \varepsilon^{j k 3} \Pi_{k} \nabla_{R_{i}} \Delta}
\end{aligned}
$$

and we obtain in addition the non trivial commutation relation between the covariant momenta,

$$
\left[\pi_{x}, \pi_{y}\right]=-i e \hbar B_{z}+i e^{2} \hbar^{2} \Theta B_{z}^{2} .
$$

We notice also that only the component of the magnetic field in the $z$-direction (perpendicular to the graphene plane) appears in the energy and in the definition of the covariant momentum. This peculiarities is due to the 2-dimensional nature of the graphene.

From the above expressions it is also natural to introduce the magnetization vector (similar to the spin)

$$
m(\pi)=-e \hbar E \Theta=\frac{e \hbar \tau \Delta}{2 E^{2}}
$$

so that interaction with the magnetic field reads $-m B_{z}$ with the magnetic moment $m$ being an intrinsic one, associated to a valley and originated from Berry curvature. The magnetic moment which interacts with the magnetic field in the $z$-direction only is an important quantity which allows to make a distinction between the carriers of the two valleys as proposed by Xiao et.al. [14. Here we will show that the magnetization is an essential quantity for the computation of the energy levels (Landau levels). 


\subsection{Semiclassical quantization of cyclotron orbit}

Thus to simplify the discussion consider a zero electric field $V(\mathbf{r})=0$, a constant lattice distortion $\Delta(\mathbf{R})=c s t=\Delta$ and a constant magnetic field $\mathbf{B}=B \mathbf{e}_{z}$.

We start with the general semiclassical equations of motion for electrons in magnetic Bloch bands

$$
\dot{\mathbf{r}}=\frac{\partial \varepsilon}{\partial \pi}-\hbar \dot{\pi} \times \boldsymbol{\Theta}, \text { and } \dot{\pi}=-e \dot{\mathbf{r}} \times \mathbf{B}
$$

where $\varepsilon$ is the energy of a magnetic Bloch band. We have an anomalous velocity contribution $\dot{\pi} \times \boldsymbol{\Theta}$ which is the "dual" of the relativistic Lorentz force $\dot{\mathbf{r}} \times \mathbf{B}$ (for a non-uniform $B$ one must add to the second equation a kind of Stern and Gerlach term $-m \nabla B$ which couples the magnetization to the gradient of the magnetic field).

Combining both equations we have

$$
\dot{\mathbf{r}}=\frac{1}{1-e \hbar \mathbf{B} \cdot \boldsymbol{\Theta}}\left(\frac{\partial \varepsilon_{\tau}}{\partial \pi}\right) \text { and } \dot{\pi}=\frac{-e}{(1-e \hbar \mathbf{B} \cdot \boldsymbol{\Theta})}\left(\frac{\partial \varepsilon_{\tau}}{\partial \pi} \times \mathbf{B}\right)
$$

These are the usual semiclassical equations of motion of an electron in a magnetic Bloch band except for the presence of the term $1-e \hbar \mathbf{B} . \boldsymbol{\Theta}$ which as $\mathbf{B}=B \mathbf{e}_{z}$ and $\boldsymbol{\Theta}=\Theta \mathbf{e}_{z}$ becomes $1-e \hbar B \Theta$. For the graphene case we have $\varepsilon=\varepsilon_{\tau}=\sqrt{\pi^{2}+\Delta^{2}}-m B$. Therefore, the equations of motion to the first order in $\hbar$ becomes

$$
\dot{\mathbf{r}}=\pi\left(\frac{1-e \hbar B \Theta}{E}\right), \quad \dot{\pi}=e \pi \times \mathbf{B}\left(\frac{1-e \hbar B \Theta}{E}\right)
$$

which are the same than for a relativistic particle in a magnetic field except for the additional multiplicative factor $1-e \hbar B \Theta$. Note that we have $\pi \frac{d \pi}{d t}=0$ so that $E$ is a constant of motion, and also $\frac{d \Theta}{d t}=0$ and $\frac{d m}{d t}=0$.

We now focus on the non-relativistic case $\Delta^{2}>>\pi^{2}$ so that $\varepsilon_{\tau} \approx \frac{\pi^{2}}{2 \Delta^{2}}+\Delta-$ $m B$ and in terms of the coordinates Eq. (31) reads

$$
\dot{x}=\pi_{x}\left(\frac{1-e \hbar B \Theta}{\Delta}\right), \quad \dot{y}=\pi_{y}\left(\frac{1-e \hbar B \Theta}{\Delta}\right)
$$

and

$$
\dot{\pi}_{x}=-e \pi_{y} B\left(\frac{1-e B \hbar \Theta}{\Delta}\right), \quad \dot{\pi}_{y}=e \pi_{x} B\left(\frac{1-e B \hbar \Theta}{\Delta}\right)
$$

Choosing the gauge $A_{x}=0$ and $A_{y}=B X=B\left(x-\tau \frac{\hbar \pi_{y}}{4 \Delta^{2}}\right)$ we have $\pi_{y}=$ $p_{y}+e B\left(x-\tau \frac{\hbar}{4 \Delta^{2}} \pi_{y}\right)+\tau \frac{e \hbar \pi_{y}}{4 \Delta^{2}} B=p_{y}+e B x$ (note the importance of the Berry connection here), which gives $\dot{p}_{y}=\dot{\pi}_{y}-e B \dot{x}=0$ thus $p_{y}$ is a constant of motion. Or as $(1-e \hbar B \Theta) / \Delta$ is a constant of motion we can write

$$
\ddot{x}=\omega^{2}\left(x_{0}-x\right)
$$


which is the equation of a harmonic oscillator with the orbit center $x_{0}=\frac{p_{y}}{-e B}$ which is the usual expression except that it is expressed in terms of the covariant coordinate and momentum and not in terms of the canonical ones. The cyclotron frequency $\omega=\frac{B e}{\Delta}(1-e \hbar B \Theta)$ is also the usual result for the quantization of a non relativistic electron in a magnetic field except that it is slightly corrected. But this correction is actually negligible for the computation of the energy levels and we have

$$
E_{n} \approx \pm\left(\frac{\hbar e B}{\Delta}\left(n+\frac{1}{2}-\frac{\tau}{2}\right)+\Delta\right)+O\left(\hbar^{2}\right)
$$

where the contribution $\frac{\tau}{2}$ comes from the magnetization term. At the semiclassical order this expression can also be written

$$
E_{n} \approx \pm \sqrt{\Delta^{2}+2 \hbar B e\left(n+\frac{1}{2}-\frac{\tau}{2}\right)}+O\left(\hbar^{2}\right)
$$

Therefore the ground state is not degenerate as there is only one possibility to realize it $n=0$ and magnetization up. We mention here the lattice distortion in the present case that leads to a mass in the Dirac equation is imposed by hand. In a very interesting paper [19], an explicit mechanism relying on an electronlattice interaction in the presence of an external magnetic field is proposed for the lattice distortion and which leads to the energy levels Eq. (36).

It is interesting to show that one can easily recover this result from the semiclassical Bohr-Sommerfeld quantification rule

$$
\oint P_{x} d X=2 \pi \hbar(n+1 / 2)
$$

From (24), $\pi=\mathbf{P}+e \mathbf{A}+\tau \frac{e \hbar \Pi}{2 E(E+\Delta)} B$, we deduce that $P_{x}=\pi_{x}-\tau \frac{e \hbar \pi_{x} B}{2 E(E+\Delta)}$ (for $A_{x}=0$, and $\boldsymbol{\Pi}_{x}$ can safely replace by $\pi_{x}$ at this order in $\hbar$ ). In the same manner (24) gives $d X=d x-\tau \frac{\hbar d \pi_{y}}{2 E(E+\Delta)}$ (as $E$ is a constant of motion). This leads to

$$
\pi^{2}\left(1-\tau \frac{e \hbar B}{E(E+\Delta)}\right)=2 \hbar e B(n+1 / 2),
$$

where $\pi^{2}=\pi_{x}^{2}+\pi_{y}^{2}$. In the case $\Delta^{2}>>\pi^{2}$, we can replace $\pi^{2}$ by $\left(E_{n}+m B\right)^{2}-$ $\Delta^{2}$ and neglect the contribution $\tau \frac{e \hbar B}{E(E+\Delta)}$, so that

$$
E_{n} \approx \sqrt{\Delta^{2}+2 \hbar B e\left(n+\frac{1}{2}-\frac{\tau}{2}\right)}
$$

and we retrieve the previous result. Note a similar derivation based on the Mikitik and Sharlai's theorem [20] about the Berry phase in the Onsager semiclassical quantization of cyclotron orbits is provided in [21].

In the opposite regime $\Delta^{2}<<\pi^{2}, \pi^{2} \approx \varepsilon_{\tau}^{2}$ and (38) therefore becomes $E_{n}^{2}-\tau e \hbar B=2 \hbar e B(n+1 / 2)$ so that the Landau levels are given by

$$
E_{n}= \pm \sqrt{2 \hbar e B\left(n+\frac{1}{2}-\frac{\tau}{2}\right)}
$$


Interestingly from this semiclassical analysis we retrieve the exact result. It is important to stress that here the contribution $\frac{\tau}{2}$ does not come from the magnetization term which is zero here but from the Berry connection in momentum $A^{\mathbf{P}}$ which in the non-relativistic case turns out to be negligible.

\section{Conclusion}

The two-dimensional carbon crystalline honeycomb structure - graphene - has created an enormous amount of activity because of its striking electronic properties. Near each of the two distinct and well separated valleys (or Dirac points) in the Brillouin zone, the energy dispersion of electrons has a linear structure with electrons moving with an (energy independent) Fermi velocity. It means that the electrons can be regarded as massless relativistic two dimensional Dirac particles. Thus a number of exotic relativistic phenomena are, in principle, observable in graphene. It is possible to simultaneously consider the two valleys in a four dimensional framework and this allows us to apply the machinery of four component Dirac electron in the planar system of graphene.

In the present paper we have exploited a generalized form [6] of the well known Foldy Wouthuysen 3 transformation to generate the energy spectrum (in a semi-classical quantization scheme). We construct the covariant position and momentum operators which, on account of the intrinsic Berry potentials, satisfy a non-commutative phase space algebra. The induced Berry curvature terms directly affect the electron dynamics, as for example by generating an anomalous velocity term.

The main advantages of employing the generalized Foldy Wouthuysen formalism [6] is twofold:

(i) Different forms of interactions can be studied in a unified way.

(ii) The approximation scheme leads in a straightforward way to semi-classical results which are thus valid for a small ratio of the electron wavelength to the typical spatial scale of external perturbations (either in mass or in external potential).

Referring to (i) above, in the present paper we have discussed effects of an arbitrary position dependent lattice distortion which gives rise to a novel "valleyorbit" coupling (similar to the spin-orbit coupling) where one observes that the lattice distortion induces an effective electric field. Obviously our general expressions (such as Berry curvature) reduce to the well known results of [14 for constant lattice defect. It is important to stress that in both cases the intrinsic inhomogeneity of the lattice drives the effect without any external electric field. We have also studied of effects external electric and magnetic fields.

In the context of (ii) above, we have shown that one can study both the "ultrarelativistic" and "non-relativistic" limits in a single setup depending on the effective mass-gap (or lattice defect $\Delta$ ) being smaller or larger than the electron momentum.

Acknowledgement 1 The authors acknowledge fruitful discussions with $F$. Piéchon and J.N. Fuchs. We thank the referees for the constructive comments. 


\section{References}

[1] K. S. Novoselov et al., Science 306 (2004) 666; V. P. Gusynin, S. G. Sharapov and J. P. Carbotte, arXiv:0706.3016.

[2] G. Semenoff, Phys. Rev. Lett. 53 (1984) 2449.

[3] L. L. Foldy and S.A.Wouthuysen, Phys.Rev. 78 (1950) 29.

[4] A. Berard and H. Mohrbach. Phys. Rev. D 69 (2004) 127701; Phys. Lett. A 352 (2006) 190

[5] P. Gosselin, A. Berard and H. Mohrbach, Phys. Rev. D 75 (2007) 084035; Europhys.Lett 76 (2006) 651, Phys.Lett. A 368 (2007) 356.

[6] P. Gosselin, A. Bérard, H. Mohrbach, Eur. Phys. J. B 58 (2007) 137; P. Gosselin, J. Hanssen, H. Mohrbach, Phys. Rev. D 77 (2008) 085008.

[7] M. V. Berry, Proc. Roy. Soc. London A 392 (1984) 45; A.Shapere and F.Wilczek, Geometric Phases in Physics, World Scientific, 1989.

[8] D. Xiao, J. Shi and Q. Niu, Phys. Rev. Lett. 95 (2005) 137204; C. Duval, Z. Horvath, P. Horvathy, L. Martina and P. Stichel, Phys. Rev. Lett. 96 (2006) 099701.

[9] K. Y. Bliokh, Europhys. Lett. 72, (2005) 7; Phys. Lett. A 351 (2006) 123.

[10] For a review, see K. Y. Bliokh and Y. P. Bliokh, Ann. Phys. (N.Y.) 319 (2005) 13.

[11] W. Greiner, Relativistic Quantum Mechanics, Springer, 1990.

[12] H. S. Snyder, Phys. Rev. 71 (1947) 68.

[13] H. Longuet-Higgins et. al., Proc. R. Soc. A 224 (1958) 1; C. Mead and D. Truhlar, J. Chem. Phys. (1979) 702284.

[14] D. Xiao, W. Yao and Q. Niu, Phys. Rev. Lett. 99 (2007) 236809.

[15] M. C. Chang et al, J. Phys.: Condens. Matter 20 (2008) 193202 .

[16] S. Murakami, N. Nagaosa, S.-C. Zhang, Science 301 (2003) 1348.

[17] J. Sinova et al., Phys Rev. Lett. 92 (2004) 126603.

[18] B. A. Bernevig and S. C. Zhang, Phys. Rev. Lett. 96, (2006) 106802.

[19] J. N. Fuchs and P. Lederer, Phys. Rev. Lett.98, (2007) 016803.

[20] G. P. Mikitik and Yu. V. Sharlai, Phys. Rev. Lett. 82, 2147 (1999).

[21] J. N. Fuchs, G. Montambaux, F. Piéchon and M. Goerbig, "Landau levels of boron nitride: Berry phase and phase mismatch", in preparation. 